\title{
ASOCIACIÓN ENTRE VALORES DE TRABAJO, INTERESES PROFESIONALES Y RASGOS DE PERSONALIDAD
}

\section{ASSOCIATIONS BETWEEN WORK VALUES, PROFESSIONAL INTERESTS AND PERSONALITY TRAITS}

\author{
Lariana Paula Pinto* \\ Ana Paula Porto Noronha** \\ Fabián Javier Marín Rueda** \\ *Universidad de São Francisco. Brasil \\ **Programa de Postgrado en Psicología, Universidad de São Francisco. Brasil \\ **CNPq. Brasil
}

\begin{abstract}
Resumen: Este estudio tuvo como objetivo investigar la asociación entre intereses profesionales y valores de trabajo, y entre valores de trabajo y rasgos de personalidad. Participaron 281 universitarios, con edad media de 23.97 años, de los cursos de Administración, Psicología, Filosofía y Teología, del Estado de San Pablo, Brasil. Fueron aplicados tres instrumentos (Escala de Evaluación de los Valores de Trabajo, Cuestionario de Búsqueda Auto-Dirigida y Batería Factorial de Personalidad). Los resultados indicaron que los factores 'Gerenciamiento y Responsabilidad' y 'Preocupación Social' de la Escala de Evaluación de los Valores de Trabajo explicaron mejor los intereses 'Realista', 'Social', 'Convencional' y 'Artístico', con varianza explicada entre $2 \%$ y $18 \%$. En relación a los rasgos de personalidad como predictores de los valores de trabajo, los resultados indicaron valores entre $9 \%$ a $33 \%$, siendo 'Competencia' la faceta más presente en los modelos. Los resultados se discuten en base a la literatura.
\end{abstract}

Palabras clave: evaluación psicológica; valores de trabajo; orientación profesional; rasgos de personalidad

\begin{abstract}
This study aimed to investigate associations between vocational interests, personality traits and work values. 281 students participated, with a mean age of 23.97, from business administration, psychology, philosophy and theology, of the state of São Paulo. Three instruments (Scale Work Values Assessment, Search Quiz Self-Addressed and Battery Personality Factor) were use. The results indicated that the social responsibility and management factors explained the interests Realistic, Social, Conventional and Artistic, with an explained variance between $2 \%$ and $18 \%$. With respect to personality traits as predictors of working values, results showed values between $9 \%$ and 33\%, with 'Competence' as the most present facet in the models. The results are discussed according to the literature.
\end{abstract}

Keywords: psychological assessment; work values; professional guides; personality traits

Debido a su relevancia en el ambiente laboral, los valores de trabajo han recibido atención de los investigadores del área de orientación profesional y de carrera, así como del área organizacional (Chernyak-Hai \& Tziner, 2016; Consiglio, Cenciotti, Borgogni, Alessandri, \& Schwartz, 2016; Sortheix, Chow, \& Salmela-Aro, 2015; Sverko, Barbarovi, \& Sverko, 2008; Tien, 2011). Especialmente en el campo de la orientación profesional, contexto del cual este estudio hace parte, la evaluación de los valores de trabajo, junto con los intereses vocacionales, son ampliamente utilizados (Sodano, 2011). Según afirman Rounds y Armstrong (2005), las comparaciones entre los valores de un individuo y las diferentes ocupaciones pueden mejorar la com- prensión sobre las funciones en que habrá mayor confort y bienestar, y también estimar la posible adaptación a determinada actividad. El uso de una medida que ofrezca informaciones sobre los valores, debe ser parte de una estrategia integrada que combine diferentes fuentes de conocimiento del individuo y lo ayude en el proceso de orientación profesional.

Super (1973) sugirió un modelo jerárquico que envuelve necesidades, valores de trabajo, rasgos de personalidad e intereses profesionales. Las necesidades son consideradas como la falta de algo que cuando aparece, contribuye para el bienestar del individuo. De esa forma, rasgos, valores e intereses derivan de las necesidades. Los rasgos son características relativas a formas 
habituales de reaccionar para atender una necesidad en una determinada situación; los valores son objetivos que se pretenden alcanzar para satisfacer una necesidad; y los intereses son las preferencias por actividades específicas u objetos por medio de los cuales los valores pueden ser alcanzados y las necesidades atendidas. En otras palabras, las necesidades son satisfechas, en la medida que los rasgos y los valores de trabajo son atendidos.

Años después, Super (1995) reafirmó la concepción de que la relación entre intereses, valores y necesidades se justifica porque los intereses se expresan por medio de las actividades que se realizan para poder alcanzar los valores, y como consecuencia de eso satisfacer sus necesidades. De esa forma, el autor presentó una asociación jerárquica entre los tres constructos. Para él, las necesidades están relacionadas a condiciones fisiológicas y a la sobrevivencia y son experimentadas como un sentimiento de falta de algo, con el deseo de llenar ese vacío. Los valores resultan de las metas establecidas para atender las necesidades, y por lo tanto, la percepción de una falta lleva a valorizar algo que parece suplirla. Por fin, los intereses son las actividades en las cuales el individuo espera concretizar sus valores.

De acuerdo con Savickas (1999), la distinción entre los intereses profesionales y los valores de trabajo puede ser observada en aspectos particulares de una ocupación a la cual el individuo valora o ve como importante, pero no elige para desarrollarla. Consecuentemente, aquellos individuos que poseen intereses semejantes pueden evaluar características específicas del mismo ambiente ocupacional de manera diferente, aunque tengan el mismo interés. Considerando eso, Sodano (2011) afirmó que los intereses pueden ser considerados como manifestaciones de valores, y por tanto, poseen algunos atributos comunes, aunque no se trate del mismo constructo. El autor destaca que los intereses se refieren a gustar o no de determinadas actividades y situaciones, mientras que los valores se refieren a la relativa importancia atribuida a ellas.

Corroborando la perspectiva de Super (1973; 1995), Vinken (2007) resalta la importancia de entender esos constructos, y especialmente incluir la evaluación de los valores de trabajo, ya que influyen mucho en el proceso de orientación de carrera. A su vez, Leuty y Hansen $(2011 ; 2013)$ resaltan que mientras que los intereses han demostrado fuerza de predicción en la elección de la carrera, los valores también están asociados a esa elección, siendo utilizados como informaciones adicionales para sus opciones de carrera. Además de eso, la relación entre valores de trabajo y rasgos de personalidad también ha sido objeto de investigaciones (Berings, Fruyt, \& Bouwen, 2004; Furnham, Petrides, Tsaousis, Pappas, \& Garrod, 2005; entre otras). De manera general, los rasgos son considerados predictores de los valores de trabajo, por la influencia que la personalidad ejerce en la percepción del individuo en relación a su ambiente.

Rounds y Armstrong (2014), tratando de proponer una manera de estudiar la asociación entre intereses, rasgos de personalidad, valores, necesidades y habilidades, establecieron relaciones entre varios modelos publicados por los propios autores. A título de síntesis, construyeron el Atlas, que consiste en una figura en la cual son dispuestos esos constructos, con la proximidad teórica y explícita por la posición en la figura. En ese sentido, en relación a los 'Valores Morales' e 'Independencia' la proximidad se dio con el tipo Realista del Self Direct Search - SDS (Holland \& Rayman, 1986); 'Utilización de las Habilidades' y 'Creatividad' están próximas de lo Artístico; el 'Valor de la Autoridad' queda más cerca de lo 'Social'; e incluso, de lo 'Convencional' y las 'Políticas de la empresa'.

En un estudio longitudinal, Sortheix, Chow y Salmela-Aro (2015) analizaron el papel de los valores de trabajo en el proceso de obtención de empleo. Cuatrocientos quince estudiantes del $9^{\circ}$ año respondieron el instrumento Careers Values Scale y el desempeño académico fue obtenido por la escuela a través de los registros de calificaciones escolares. Los resultados revelaron asociación entre los valores de trabajo y autonomía, oportunidad de toma de decisión y formación profesional. Los valores de trabajo fueron asociados al desempeño académico y al desempeño en el trabajo (autonomía, proceso de decisión y formación profesional) en diferentes momentos del estudio longitudinal. Por fin, los resultados verificaron la idea de que los valores de trabajo son importantes antecedentes de éxito en la transición para el empleo.

Con la intención de relacionar intereses profesionales y valores de trabajo a los rasgos de personalidad, Duffy, Borges y Hartung (2009) analizaron las respuestas dadas por 282 estudiantes de medicina a los instrumentos Physician Values in Practice Scale, NEO-PI-R y Strong Interest Inventory. Los valores evaluados corresponden a seis factores: Prestigio, Servicio, Autonomía, Estilo de vida, Gerenciamiento y Actividades Académicas. En lo referente a los resultados de las correlaciones, los valores de trabajo presentaron coeficientes negativos, magnitud baja y significativa, sólo con 
los intereses de investigación relacionados al valor Actividades Académicas; así como las actividades de la dimensión Social con el valor Prestigio. En relación a la personalidad, apenas el rasgo Amabilidad se correlacionó significativa y negativamente con el valor Prestigio. Cuando fue analizada la predicción de los valores de trabajo, éstos se mostraron buenos predictores para los intereses vocacionales, con una variancia de $10 \%$ para el tipo Social, $14 \%$ para el tipo Investigativo y $6 \%$ para el tipo Convencional. En el caso de los rasgos de personalidad, Apertura y Extraversión revelaron 3\% de variancia, Amabilidad 8\%, e Inestabilidad Emocional 2\%.

Considerando los señalamientos teóricos y las investigaciones realizadas, articular las relaciones entre valores de trabajo y otras variables proporcionan informaciones que pueden mejorar el proceso de orientación profesional y de carrera. Tal mejora puede ocurrir al promover una mejor comprensión de los diferentes mecanismos utilizados por el sujeto en su elección o re-opción ocupacional, para hacer uso de información de características integradas de personalidad, intereses profesionales y valores de trabajo. En Brasil, todavía son muy pocas las publicaciones que recurren a los valores de trabajo para investigar las delimitaciones y relaciones con otras variables, o como fuente de información en el proceso de orientación profesional y de carrera. El objetivo de este estudio fue analizar la asociación entre valores de trabajo, intereses y rasgos de personalidad. Se investigó qué rasgos de personalidad contribuyen mejor a la predicción de los valores de trabajo y si éstos poseen poder predictivo en relación a los intereses profesionales.

\section{Método}

\section{Participantes}

Participaron en el estudio 281 universitarios, estudiantes de los cursos de Administración $(28 ; 9.9 \%)$, Psicología (190; 67.6\%), Filosofía (34; $12.1 \%$ ) y Teología $(29 ; 10.4 \%)$, del estado de San Pablo, Brasil. Del total, 101 (35.9\%) eran del sexo masculino y $180(64.1 \%)$ del femenino, con edad entre 18 a 56 años $(M=23.97 ; D E=6.82)$.

\section{Instrumentos}

- Escala para Evaluación de los Valores de Trabajo (EAVT), (Pinto \& Noronha, 2014).

Compuesta por 65 ítems en escala Likert de cinco puntos, que va de 1 (mínimo - nada importante) a 5 (máximo - muy importante). Los ítems fueron construidos con base en las 15 dimensiones propuestas por Super (1957), y después del análisis de la estructura interna esas dimensiones fueron aglomeradas, por extracción del eje principal y rotación direct oblimin, en cuatro factores. El primer factor, denominado 'Reconocimiento y Estabilidad', está compuesto por 30 ítems que representan valores relacionados a credibilidad y prestigio por medio de la actividad profesional, así como la garantía de mantenerse financieramente estable, y seguro en el ambiente de trabajo. El factor siguiente, 'Desafío y Autonomía', se compone por 18 ítems que tratan de acciones desafiantes y que exigen búsqueda continua de conocimiento, al mismo tiempo que requieren independencia en la ejecución de las tareas. Con 12 ítems, el factor llamado 'Gerenciamiento y Responsabilidad' corresponde a la valoración de la supervisión de actividades desempeñadas por subordinados, o de la elaboración del producto final de cualquier naturaleza. El cuarto factor, 'Preocupación Social', con 5 ítems, se refiere a la valoración de la contribución que la actividad profesional puede ofrecer a la sociedad, promoviendo mejorías y beneficios. En lo que respecta a las estimaciones de precisión, el análisis de consistencia interna reveló excelentes coeficientes, que van de 0.84 a 0.93 .

- Cuestionario de Búsqueda Auto-Dirigida (Self-Direct Search, SDS), (Primi, Mansão, Muniz, \& Nunes, 2010).

El instrumento fue desarrollado con el objetivo de verificar los intereses profesionales, teniendo como base los seis tipos del modelo hexagonal de John Holland: Realista (R), Investigativo (I), Artístico (A), Social (S), Emprendedor (E) y Convencional (C). Los tipos describen aspectos como rasgos de personalidad, objetivos de vida, valores, identificaciones, competencias y habilidades. Está estructurado en cuatro secciones: actividades, competencias, carreras y habilidades. Las tres primeras son compuestas por 66 ítems cada una y son presentadas en estilo dicotómica - S (sí, 1) o $\mathrm{N}$ (no, 0). La sección Habilidades posee 12 ítems, tipo Likert de siete puntos, que van de 1 (baja habilidad) hasta 7 (alta habilidad). Con relación a los estudios de validez y precisión, Primi y cols. (2010), al analizar la dimensionalidad del SDS, encontraron los seis tipos de intereses propuestos por Holland. Con relación al análisis de la consistencia interna, los coeficientes variaron de 0.90 a 0.94 y la estabilidad temporal fue medida después de tres meses, con coeficientes entre 0.76 y 0.89 . Para el presente estudio, fue utilizada sólo la prueba referente a la sección Actividades. 
- Batería Factorial de Personalidad (BFP) (Nunes, Hutz, \& Nunes, 2010).

La Batería tiene por finalidad evaluar la personalidad a partir del modelo de los Cinco Grandes Factores (CGF), que incluye las dimensiones Extraversión (25 ítems), Sociabilidad (28 ítems), Responsabilidad (21 ítems), Inestabilidad Emocional (29 ítems) y Apertura a Experiencias (23 ítems). Está compuesta por 126 ítems en escala tipo Likert de 7 puntos, que varían desde 'me describe muy mal' a 'me describe muy bien'. Estudios de fiabilidad fueron realizados y los valores de alfa encontrados variaron de 0.74 a 0.89 . Entre los estudios de validez, fue realizado el análisis de estructura interna y fue encontrada la solución factorial de cinco factores, como base teórica del instrumento. Los eingenvalues variaron de 4.59 a 10.04 . El instrumento también presenta evidencias de validez basadas en la relación con otros constructos, como por ejemplo, intereses profesionales, inteligencia, bienestar subjetivo, entre otros (Nunes, et al., 2010).

\section{Procedimientos}

La investigación fue iniciada después de la autorización de las cuatro instituciones de donde los datos serían recogidos y de la aprobación del Comité de Ética en Investigación de la Universidad de São Francisco. Los instrumentos fueron aplicados colectivamente, en clase, y todos los datos fueron recolectados por los autores. En cuanto al orden de aplicación de los instrumentos, la EAVT fue siempre la primera en ser respondida, seguida de la BFP y del SDS. No todos los participantes respondieron los tres instrumentos, por lo tanto las aplicaciones fueron en tres bloques, siendo el primer bloque aquel cuyos alumnos respondieron la EAVT y el SDS, en el segundo fueron respondidas la EAVT y la BFP, y finalmente, el tercer bloque en el cual los tres tests fueron realizados. En este último caso, el orden del segundo y tercer test utilizado fue alternado, con la intención de minimizar el efecto cansancio. Las aplicaciones duraron arpximadamente de 60 minutos.

\section{Plan de Análisis de Datos}

Para atender los objetivos propuestos en este estudio se recurrió al método de análisis de regresión por etapas (stepwise), con el fin de revelar qué variables, en el caso de los rasgos de personalidad, contribuyen mejor en la predicción de los valores de trabajo, a medida que significativamente son agregados al modelo (Hair et al., 2009). También se verificó si los valores de trabajo (variables independientes) poseen algún poder predictivo en relación a los intereses profesionales (variables dependientes). Para ambos análisis de regresión, se examinó el valor del factor de variancia de la inflación (VIF) que ofrece información de multicolinealidad, que indica si un pronosticador tiene una fuerte relación lineal con la otra. Los valores de VIF obtenidos fueron inferiores a 10, considerados favorables para conducir la regresión (Hair et al., 2009).

\section{Resultados}

Primero son presentados los estadísticos descriptivos de los instrumentos utilizados, y como se puede observar en la tabla 1, sobre la EAVT, el valor de trabajo que más apareció fue Reconocimiento y Estabilidad $(M=4.46 ; D E=0.46)$, mientras que Gerenciamiento y Responsabilidad presentó el promedio más bajo $(\mathrm{M}=3.33 ; D E=0.53)$. En relación a los intereses profesionales, las actividades de la dimensión Social también fueron las más presentes en la muestra $(M=9,06 ; D P=2,06)$, y las menos presentes fueron las actividades de la dimensión Convencional $(M=2,56 ; D P=3,13)$. En los rasgos de personalidad medidos por la BFP, se observó que Sociabilidad fue el más presente, con una puntuación promedio de $5.33(D E=0.61)$, mientras que Instabilidad emocional fue el menos presente $(M=3.27 ; D E=0.93)$.

Para investigar evidencias de validez basadas en constructos relacionados a la Escala de Evaluación de los Valores de Trabajo (EAVT), se recurrió al análisis de regresión. Fueron utilizados los resultados obtenidos en la EAVT junto con los referentes a la Batería Factorial de Personalidad (BFP) y el Cuestionario de Búsqueda Auto-Dirigida (Self-Direct Search, SDS). Inicialmente, los coeficientes del método de análisis de regresión por etapas (stepwise) entre los valores de trabajo de la EAVT y los intereses profesionales, medidos por el SDS, serán presentados en la tabla 2. Cabe destacar que se probó multicolinealidad, no habiendo sido identificadas violaciones, ya que los valores de VIF fueron inferiores a 10, siendo adecuados, conforme sugieren Hair et al. (2010).

Analizando la Tabla 2 se observa la formación de modelos predictores y significativos sólo en relación a tres tipos del SDS, Artístico, Social y Emprendedor; mientras que los demás tipos no revelaron relación predictiva a partir de los valores de trabajo. El primer análisis reveló formación del modelo Interés Artístico previsto por el factor 
Preocupación Social $(\beta=0.160)$, cuya variancia explicada fue pequeña $\left(R^{2}=0.02\right)$. Fue revelada una leve correlación entre ellos, y lo mismo ocurrió en relación a la regresión, de modo que la importancia atribuida a los buenos puestos de operaciones realizadas para la sociedad, mínimamente presupone la preferencia por actividades que favorezcan la expresión individual y el uso de la creatividad. Con una variancia explicada final de $6 \%\left(R^{2}=0.06\right)$, el tipo Social fue predicho por dos valores de trabajo, Preocupación Social $(\beta=0.241)$, y Gerenciamiento y Responsabilidad $(\beta=-0.203)$. Esas informaciones indican que personas que valoran la ejecución de tareas en favor de la sociedad, y no dan mucha importancia a supervisar la tarea de otras personas, prefieren actividades que se relacionan más con las interacciones sociales. El modelo que evidenció mayor capacidad predictiva fue el tipo Emprendedor, que tuvo su variancia explicada en $18 \%\left(R^{2}=0.18\right)$ por el valor de trabajo Gerenciamiento y Responsabilidad $(\beta=0.429)$. Las personas que valoran el comando de un equipo o de un conjunto de tareas y sus efectos, tienden a ser aquellas que prefieren actividades que requieren habilidades de liderazgo y persuasión, de manera que van a ocupar funciones jerárquicamente superiores a los demás.

Seguidamente se verificó si los rasgos de personalidad (variables independientes) anteceden la valorización de determinados aspectos del trabajo (variables dependientes). Los resultados de las regresiones en el primer factor de la EAVT son presentadas en la tabla 3.

A partir de tabla 3, considerando el factor Reconocimiento y Estabilidad como el factor que se predijo, se observa la formación de dos modelos. En el modelo 1 la variable independiente que mejor predice fue la faceta R1 (Competencia), del factor Responsabilidad, que trata de características de

Tabla 1.

Estadisticos descriptivos y alfa de Cronbach de los instrumentos EAVT, SDS y BFP

\begin{tabular}{|c|c|c|c|c|c|c|}
\hline Instrumentos & $N$ & Mín. & Máx. & $M$ & $D E$ & Alfa \\
\hline \multicolumn{7}{|l|}{ EAVT } \\
\hline Reconocimiento y estabilidad & 281 & 1.14 & 5.00 & 4.46 & 0.46 & 0.92 \\
\hline Desafío y Autonomía & 281 & 1.19 & 5.00 & 4.18 & 0.53 & 0.89 \\
\hline Gerenciamiento y Responsabilidad & 281 & 1.33 & 4.91 & 3.33 & 0.63 & 0.83 \\
\hline Preocupación social & 281 & 1.00 & 5.00 & 4.40 & 0.63 & 0.84 \\
\hline \multicolumn{7}{|l|}{ SDS } \\
\hline Realista & 174 & 0 & 11 & 2.64 & 2.26 & 0.76 \\
\hline Investigativo & 175 & 0 & 11 & 6.36 & 2.82 & 0.78 \\
\hline Artístico & 175 & 0 & 11 & 6.83 & 2.81 & 0.78 \\
\hline Social & 175 & 3 & 11 & 9.06 & 2.06 & 0.72 \\
\hline Emprendedor & 175 & 0 & 11 & 5.38 & 2.58 & 0.74 \\
\hline Convencional & 175 & 0 & 11 & 2.56 & 3.13 & 0.89 \\
\hline \multicolumn{7}{|l|}{ BFP } \\
\hline Instabilidad emocional & 152 & 1.26 & 5.51 & 3.27 & 0.93 & 0.90 \\
\hline Extraversión & 152 & 1.68 & 6.24 & 4.26 & 0.91 & 0.88 \\
\hline Sociabilidad & 152 & 3.63 & 6.83 & 5.33 & 0.61 & 0.77 \\
\hline Responsabilidad & 152 & 2.35 & 6.80 & 4.89 & 0.87 & 0.86 \\
\hline Apertura a experiencias & 152 & 2.54 & 6.27 & 4.58 & 0.68 & 0.70 \\
\hline
\end{tabular}

Tabla 2.

Análisis de regresión jerárquica entre los intereses profesionales del modelo de Holland y los valores de trabajo

\begin{tabular}{|c|c|c|c|c|c|c|c|c|}
\hline \multirow{2}{*}{$\begin{array}{c}\text { Dimensiones } \\
\text { SDS }\end{array}$} & \multirow[t]{2}{*}{ Mod. } & \multirow{2}{*}{$\begin{array}{l}\text { Predictores } \\
\text { Factores } \\
\text { EAVT }^{1} \\
\end{array}$} & \multicolumn{2}{|c|}{$\begin{array}{c}\text { Coeficientes } \\
\text { estandarizados }\end{array}$} & \multirow{2}{*}{$\begin{array}{c}\begin{array}{c}\text { Coeficientes no } \\
\text { estandarizados }\end{array} \\
B \\
\end{array}$} & \multirow[t]{2}{*}{$R^{2}$} & \multirow{2}{*}{$\begin{array}{c}F \\
(g l, n-g l)\end{array}$} & \multirow[t]{2}{*}{$p$} \\
\hline & & & $B$ & $E P$ & & & & \\
\hline \multirow[t]{2}{*}{ Artístico } & 1 & (Const.) & 3.803 & 1.432 & - & \multirow[b]{2}{*}{0.02} & 4.562 & \multirow{2}{*}{$*$} \\
\hline & & Factor 4 & 0.702 & 0.329 & 0.160 & & (1.173) & \\
\hline \multirow[t]{5}{*}{ Social } & 1 & (Const.) & 6.666 & 1.048 & & \multirow{2}{*}{0.02} & 5.318 & \multirow{2}{*}{$*$} \\
\hline & \multirow{4}{*}{2} & Factor 4 & 0.555 & 0.241 & 0.173 & & (1.173) & \\
\hline & & (Const.) & 8.040 & 1.160 & - & \multirow{3}{*}{0.06} & $\begin{array}{c}(1.1 / 3) \\
6.100\end{array}$ & \multirow{3}{*}{$*$} \\
\hline & & Factor 4 & 0.775 & 0.251 & 0.241 & & 0.100 & \\
\hline & & Factor 3 & -0.712 & 0.275 & -0.203 & & $(2.172)$ & \\
\hline \multirow[t]{2}{*}{ Emprendedor } & 1 & (Const.) & -0.779 & 1.000 & - & \multirow{2}{*}{0.18} & 39.104 & \multirow{2}{*}{$*$} \\
\hline & & Factor 3 & 1.887 & 0.302 & 0.429 & & (1.173) & \\
\hline
\end{tabular}

${ }^{1}$ Factor 3= Gerenciamiento y Responsabilidad; Factor 4= Preocupación Social. ${ }^{*} p<0.05$ 
Tabla 3.

Análisis de regresión jerárquica entre el valor de trabajo "Reconocimiento y Estabilidad" y las facetas de los rasgos de personalidad

\begin{tabular}{|c|c|c|c|c|c|c|c|}
\hline \multirow[t]{2}{*}{ Modelo } & \multirow{2}{*}{$\begin{array}{c}\text { Predictores } \\
\text { Factores BFP }\end{array}$} & \multicolumn{2}{|c|}{$\begin{array}{c}\text { Coeficientes } \\
\text { estandarizados }\end{array}$} & \multirow{2}{*}{$\begin{array}{c}\begin{array}{l}\text { Coeficientes no } \\
\text { estandarizados }\end{array} \\
B\end{array}$} & \multirow[t]{2}{*}{$R^{2}$} & \multirow{2}{*}{$\begin{array}{c}F \\
(g l, n-g l)\end{array}$} & \multirow{2}{*}{$p$} \\
\hline & & $B$ & $E P$ & & & & \\
\hline \multirow[t]{2}{*}{1} & (Const.) & 116.523 & 6.109 & - & \multirow{2}{*}{0.05} & 8.639 & \multirow{2}{*}{$<0.05$} \\
\hline & $\mathrm{R} 1$ & 3.527 & 1.200 & 0.233 & & $(1.150)$ & \\
\hline 2 & $\begin{array}{l}\text { (Const.) } \\
\text { R1 } \\
\text { N2 }\end{array}$ & $\begin{array}{c}104.880 \\
4.282 \\
2.222\end{array}$ & $\begin{array}{l}7.425 \\
1.210 \\
0.837\end{array}$ & $\begin{array}{l}0.283 \\
0.213\end{array}$ & 0.09 & $\begin{array}{c}8.015 \\
(2.149)\end{array}$ & $<0.05$ \\
\hline
\end{tabular}

R1= Competencia $;$ N2=Inestabilidad emocional

personas que poseen una percepción favorable de sí mismas y creen en su capacidad, y que explica sólo el 5\% de la variancia. Para el modelo 2 se incluyó la faceta N2 (Inestabilidad emocional) del factor Neuroticismo, siendo la variancia explicada de $9 \%$, de manera que fueron introducidos los aspectos que describen cierta variabilidad de humor. De esa forma, esas facetas contribuyen de forma mínima con la predicción de los valores relacionados a la necesidad de tener su trabajo reconocido por la sociedad, así como también la búsqueda por mantenerlo. Teniendo en vista los modelos formados para el primer factor de la EAVT, la tabla 4 presenta los modelos encontrados para el segundo factor, Desafío y Autonomía.

Analizando la tabla 4 se observa la formación de 5 modelos predictivos en relación al factor Desafío y Autonomía. En el primer modelo, la faceta adicionada fue R1 (Competencia) del factor Responsabilidad, explicando $21 \%$ de la variancia, y se refiere a comportamientos de personas activas que buscan sus objetivos y tienen conciencia de su competencia en realizar actividades difíciles. En seguida, se nota que la explicación pasa a ser de $28 \%$ con la introducción de la faceta S1 (Amabilidad), del rasgo Sociabilidad, que trata de personas atentas y con interés de auxiliar a los demás. El modelo siguiente explicó $30 \%$, teniendo participación de características de individuos que no son cuidadosos al expresar sus ideas y no saben evaluar las consecuencias de sus acciones, descriptas por la faceta R2 (Ponderación-Prudencia). Se debe destacar que esas características representan puntuaciones bajas en la faceta, ya que el coeficiente de regresión negativo $(\beta=-0.161)$ indica esa relación inversa.

Tabla 4.

Análisis de regresión jerárquica entre el valor de trabajo "Desafio y Autonomía” y las facetas de los rasgos de personalidad

\begin{tabular}{|c|c|c|c|c|c|c|c|}
\hline \multirow[t]{2}{*}{ Modelo } & \multirow{2}{*}{$\begin{array}{c}\text { Predictores } \\
\text { Factores BFP }\end{array}$} & \multicolumn{2}{|c|}{$\begin{array}{c}\text { Coeficientes } \\
\text { estandarizados }\end{array}$} & \multirow{2}{*}{$\begin{array}{c}\begin{array}{l}\text { Coeficientes no } \\
\text { estandarizados }\end{array} \\
B\end{array}$} & \multirow[t]{2}{*}{$R^{2}$} & \multirow[t]{2}{*}{$F(g l, n-g l)$} & \multirow[t]{2}{*}{$p$} \\
\hline & & $B$ & $E P$ & & & & \\
\hline \multirow[t]{2}{*}{1} & (Const.) & 49.735 & 3.556 & - & \multirow[b]{2}{*}{0.21} & 41.474 & \multirow{2}{*}{$<0.05$} \\
\hline & $\mathrm{R} 1$ & 4.499 & 0.699 & 0.465 & & $(1.150)$ & \\
\hline \multirow[t]{3}{*}{2} & (Const.) & 35.873 & 4.982 & - & \multirow{3}{*}{0.28} & \multirow{3}{*}{$\begin{array}{l}29.874 \\
(2.149)\end{array}$} & \multirow{3}{*}{$<0.05$} \\
\hline & R1 & 3.318 & 0.737 & 0.343 & & & \\
\hline & $\mathrm{S} 1$ & 3.526 & 0.925 & 0.291 & & & \\
\hline \multirow[t]{4}{*}{3} & (Const.) & 38.788 & 5.086 & - & \multirow{4}{*}{0.30} & \multirow{4}{*}{$\begin{array}{l}22.119 \\
(3.148)\end{array}$} & \multirow{4}{*}{$<0.0$} \\
\hline & R1 & 3.756 & 0.753 & 0.389 & & & \\
\hline & $\mathrm{S} 1$ & 3.639 & 0.914 & 0.300 & & & \\
\hline & $\mathrm{R} 2$ & -1.144 & 0.511 & -0.161 & & & \\
\hline \multirow[t]{5}{*}{4} & (Const.) & 33.115 & 5.779 & - & \multirow{5}{*}{0.31} & \multirow{5}{*}{$\begin{array}{l}17.926 \\
(4.147)\end{array}$} & \multirow{5}{*}{$<0.05$} \\
\hline & R1 & 3.757 & 0.746 & 0.389 & & & \\
\hline & $\mathrm{S} 1$ & 3.479 & 0.909 & 0.287 & & & \\
\hline & R2 & -1.452 & 0.529 & -0.204 & & & \\
\hline & $\mathrm{S} 2$ & 1.442 & 0.721 & 0.143 & & & \\
\hline \multirow[t]{6}{*}{5} & (Const.) & 26.910 & 6.311 & - & \multirow{6}{*}{0.33} & \multirow{6}{*}{$\begin{array}{l}15.799 \\
(5.146)\end{array}$} & \multirow{6}{*}{$<0.05$} \\
\hline & R1 & 3.616 & 0.738 & 0.374 & & & \\
\hline & $\mathrm{S} 1$ & 3.012 & 0.919 & 0.248 & & & \\
\hline & R2 & -1.358 & 0.523 & -0.191 & & & \\
\hline & S2 & 1.924 & 0.742 & 0.191 & & & \\
\hline & A3 & 1.413 & 0.618 & 0.165 & & & \\
\hline
\end{tabular}


El cuarto modelo incluye la faceta S2 (Prosociabilidad), nuevamente del factor Sociabilidad, que se refiere a comportamientos de riesgo, con un aumento de la predicción de $1 \%$. Además de las cuatro facetas descriptas, el último modelo revelado tuvo la participación de la faceta A3 (Búsqueda de Novedades) del factor Apertura, contando con atributos de personas que están siempre interesadas en experimentar nuevas situaciones. El modelo final tuvo 33\% de variancia explicada. De manera general, se verifica coherencia entre las facetas incorporadas al modelo final, y presentan predicción para el factor Desafío y Autonomía, al resaltar el valor por la realización de actividades que provocan estimulación intelectual y búsqueda constante de conocimiento, además de ofrecer libertad para ejecutar las tareas. La tabla 5 presenta las predicciones en relación al tercer factor de la EAVT, Gerenciamiento y Responsabilidad.

Basado en la tabla 5, el primer modelo contó con la inserción de la faceta E2 (Altivez) del factor Extraversión, y el siguiente con la faceta R1 (Competencia), cuyas predicciones fueron de 10\% y $13 \%$ de variancia explicada, respectivamente. Tales modelos sugieren que características de personas que poseen capacidad para actividades de liderazgo y persuasión, asociadas a confianza en su capacidad para realizar varias tareas al mismo tiempo, contribuyen con la predicción de los valores relacionados a las actividades que requieren estilos de liderazgo para supervisión de otras personas o tareas y compromiso con los resultados. La faceta Competencia estuvo presente como predictora de los tres primeros factores de EAVT, revelando que claridad y confianza en el planeamiento de las propias metas presupone la valorización de los aspectos tratados en esos factores. La tabla 6 presenta los resultados obtenidos en el cuarto factor de la EAVT, Preocupación Social.

Así como en los factores ReconocimientoEstabilidad y Gerenciamiento-Responsabilidad, se formaron 2 modelos para el valor de trabajo Preocupación Social, cuyos ítems representan el interés en promover ganancias y beneficios para la sociedad en general. El primer modelo reveló $22 \%$ de la variancia explicada por la faceta S1 (Amabilidad), que representa personas empáticas y comprensivas, a las que les importan las necesidades ajenas. El segundo modelo presentó explicación de $37 \%$ de variancia, siendo agregada la faceta E3 (Dinamismo) del rasgo Extraversión, que representa comportamientos de toma de iniciativas y participación en diversas actividades simultáneamente.

Tabla 5.

Análisis de regresión jerárquica entre el valor de trabajo "Gerenciamiento y Responsabilidad" y las facetas de los rasgos de personalidad

\begin{tabular}{|c|c|c|c|c|c|c|c|}
\hline \multirow[t]{2}{*}{ Modelo } & \multirow{2}{*}{$\begin{array}{l}\text { Predictores } \\
\text { Factores BFP }\end{array}$} & \multicolumn{2}{|c|}{$\begin{array}{c}\text { Coeficientes } \\
\text { estandarizados }\end{array}$} & \multirow{2}{*}{$\begin{array}{c}\text { Coeficientes no } \\
\text { estandarizados } \\
B\end{array}$} & \multirow[t]{2}{*}{$R^{2}$} & \multirow[t]{2}{*}{$F(g l, n-g l)$} & \multirow[t]{2}{*}{$P$} \\
\hline & & $B$ & $E P$ & & & & \\
\hline \multirow[t]{2}{*}{1} & (Const.) & 31.237 & 2.158 & - & \multirow{2}{*}{0.10} & 17.277 & \multirow{2}{*}{$<0.05$} \\
\hline & E2 & 2.455 & 0.591 & 0.321 & & $(1.150)$ & \\
\hline 2 & $\begin{array}{c}\text { (Const.) } \\
\text { E2 } \\
\text { R1 }\end{array}$ & $\begin{array}{c}22.899 \\
2.340 \\
1.745\end{array}$ & $\begin{array}{l}3.891 \\
0.582 \\
0.683\end{array}$ & $\begin{array}{l}0.306 \\
0.195\end{array}$ & 0.13 & $\begin{array}{l}12.221 \\
(2.149)\end{array}$ & $<0.05$ \\
\hline
\end{tabular}

E2=Altivez; $R 1=$ Competencia .

Tabla 6.

Análisis de regresión jerárquica entre el valor de trabajo "Preocupación social" y las facetas de los rasgos de personalidad

\begin{tabular}{|c|c|c|c|c|c|c|c|}
\hline \multirow[t]{2}{*}{ Modelo } & \multirow[t]{2}{*}{$\begin{array}{c}\text { Predictores } \\
\text { Factores BFP }\end{array}$} & \multicolumn{2}{|c|}{$\begin{array}{c}\text { Coeficientes } \\
\text { estandarizados }\end{array}$} & \multirow{2}{*}{$\begin{array}{c}\begin{array}{c}\text { Coeficientes } \\
\text { no } \\
\text { estandarizados }\end{array} \\
B\end{array}$} & \multirow[t]{2}{*}{$R^{2}$} & \multirow[t]{2}{*}{$F(g l, n-g l)$} & \multirow[t]{2}{*}{$P$} \\
\hline & & $B$ & $E P$ & & & & \\
\hline \multirow[t]{2}{*}{1} & (Const.) & 12.989 & 1.500 & - & \multirow[b]{2}{*}{0.22} & 43.118 & \multirow[b]{2}{*}{$<0.05$} \\
\hline & $\mathrm{S} 1$ & 1.742 & 0.265 & 0.473 & & (1.150) & \\
\hline 2 & $\begin{array}{c}\text { (Const.) } \\
\text { S1 } \\
\text { E3 }\end{array}$ & $\begin{array}{l}11.758 \\
1.416 \\
0.651 \\
\end{array}$ & $\begin{array}{l}1.486 \\
0.271 \\
0.183\end{array}$ & $\begin{array}{l}0.384 \\
0.262 \\
\end{array}$ & 0.27 & $\begin{array}{l}29.559 \\
(2.149)\end{array}$ & $<0.05$ \\
\hline
\end{tabular}

S1=Amabilidad $;$ E3=Dinamismo 


\section{Discusión}

El objetivo del estudio fue analizar si los valores son variables que predicen los intereses, así como cuáles son los rasgos de personalidad que más contribuyen en la predicción de los valores de trabajo. Para eso, se hicieron análisis de regresión con el método stepwise. En relación al primer análisis, los valores de trabajo fueron establecidos como predictores de los intereses profesionales, y los resultados encontrados revelaron poder predictivo de dos factores de la EAVT, Gerenciamiento y Responsabilidad, que reúne personas que valorizan la supervisión de las tareas y su ejecución, y Preocupación Social, que se refiere al deseo de contribuir con la mejoría y armonía de la sociedad, en relación a los tipos Artístico, Social y Emprendedor, revelando variancia explicada de dos de ellos, 6 y $18 \%$, respectivamente. Los resultados muestran coherencia teórica y replican parcialmente los hallazgos de Berings et al. (2004). La investigación de los autores evidenció capacidad predictiva de los valores, cuya variancia explicada fue de $23 \%$ para el tipo Social y $24 \%$ para el tipo Emprendedor. En relación a las diferencias entre los resultados, además de que los intereses han contribuido para explicar los valores (Artístico, Social y Emprendedor), fueron observadas algunas diferencias en cuanto a los valores más bajos de predicción, lo que se justifica en razón del menor número de medidas de valores de trabajo.

Aún en relación a lo dicho, otro estudio que tuvo un objetivo semejante fue el de Rottinghaus y Zytowski (2006), en el cual los intereses profesionales se mostraron predichos por los valores, con una variancia explicada entre 2 a $6 \%$. En relación a la investigación de Rounds y Armstrong (2014), los resultados encontrados no coincidieron, mientras que en el presente estudio, los factores 3 (Gerenciamiento y Responsabilidad) y 4 (Preocupación Social) de la EAVT estuvieron presentes en los tres modelos para los tipos Artístico, Social y Emprendedor. El factor 3 evidencia la valoración de actividades que requieren supervisar a otras personas o tareas, así como el cuidado con el resultado final del trabajo, mientras que el factor 4 se relaciona con los valores de trabajo que promueven beneficios y mejorías a la sociedad en general. Así, las diferencias están volcadas a los valores que explican mejor los tipos profesionales. En el estudio de los autores, hay más factores y ellos explican intereses distintos. Los intereses comunes obtenidos en ambos estudios fueron Artístico y Social, y hay cierta similitud entre Políticas de la Empresa y Autoridad del Instrumento utilizado por Rottinghaus y Zytowski (2006).
La relación jerárquica entre los valores de trabajo y los rasgos de personalidad también fueron verificadas, considerando el segundo constructo como predictor del primero. Los análisis indicaron capacidad predictiva de determinadas facetas de la Batería Factorial de Personalidad, en relación a los cuatro valores de trabajos medidos por la EAVT. El factor 2 (Desafío y Autonomía) fue explicado por 5 facetas, con $33 \%$ de varianza explicada, el mayor valor. Le sigue el factor 4 (Preocupación Social), con índice de 27\%. Los factores 1 (Reconocimiento y Estabilidad) y 3 (Gerenciamiento y Responsabilidad), en su orden, tuvieron valores bajos, $9 \%$ y $13 \%$ respectivamente. En común los dos últimos factores incluyen la aprobación del otro, sea el superior o el supervisado. Se puede sugerir la hipótesis de que la gratificación inmediata, sin pasar por la aprobación de otra persona se relaciona más fuertemente a los rasgos de personalidad. De modo general, las relaciones predictivas de los rasgos en relación a los valores de trabajo, fueron confirmadas por Berings et al. (2004).

Para Duffy et al. (2009) la relación predictiva también fue encontrada, con destaque para cuatro rasgos de personalidad, Inestabilidad Emocional, Extraversión, Apertura a Experiencias, y Amabilidad. Confirmando los resultados a partir de los análisis de regresión de Duffy et al. (2009), el presente estudio reveló también relación predictiva de facetas con esos factores. De manera general, se puede probar que los análisis realizados por Berings et al. (2004) y Duffy et al. (2009) fueron corroborados, verificando buenos resultados en lo que respecta a las relaciones entre los constructos investigados.

Como discusiones finales, se resalta que el objetivo de este estudio es comprender las relaciones entre intereses profesionales, valores de trabajo y rasgos de personalidad, lo que se justifica en razón de la contribución que tales asociaciones pueden tener sobre las elecciones profesionales de los individuos, una vez que las comparaciones entre los constructos a la vista de las diferentes ocupaciones pueden traer mayor confort y bienestar, así como estimar la posible adaptación a determinada actividad profesional (Leuty \& Hansen, 2011; 2013). Los investigadores, se han dedicado a estudiar el tema y sus resultados han propiciado el avance del área en la cual la temática está inserta (Berings, et al., 2004; Furnham, et al., \& Garrod, 2005; Leuty, 2013).

Las asociaciones previstas teóricamente entre valores e intereses y valores y rasgos fueron replicadas, proporcionando evidencias de validez al instrumento (Chernyak-Hai \& Tziner, 2016; Consiglio, et al., 2016; Sortheix, et al., 2015; Sverko, et al., 
2008; Tien, 2011). Mientras tanto, hay que resaltar que fueron encontradas diferencias en cuanto a predicción de factores de la Escala de Evaluación de Valores de Trabajo (EAVT) especialmente referidas a los intereses.

Como fue afirmado anteriormente, el menor número de factores de la EAVT en relación a los instrumentos utilizados en otras investigaciones puede ser parcialmente responsable por las diferencias. Los estudios mencionados son extranjeros y revelan naturalezas culturales distintas, lo que puede ser un factor que explique los datos. Por fin, no se puede olvidar de mencionar la muestra utilizada, que incluye un único estado de la federación, sin la pretensión de estratificar.

A título de agenda de investigación, además de ampliación de la muestra, sería oportuno estudiar el instrumento en relación a variables distintas como por ejemplo, sexo, edad y curso universitario. A este respecto, Sortheix, et al. (2015) incluyeron en sus estudios el status socio-económico y el desempeño académico.

\section{Referencias}

Berings, D., Fruyt, F. D., \& Bouwen, R. (2004). Work values and personality traits as predictors of enterprising and social vocational interests. Personality and Individual Differences, 36, 349-364. doi:10.1016/S0191-8869(03)00101-6

Chernyak-Hai, L., \& Tziner, A. (2016). The "I believe" and the "I invest" of Work-Family Balance: The indirect influences of personal values and work engagement via perceived organizational climate and workplace burnout. Journal of Work and Organizational Psychology, 32, 1-10. doi: 10.1016/j.rpto.2015.11.004

Consiglio, C., Cenciotti, R., Borgogni, L., Alessandri, G., \& Schwartz, S. H. (2016). The WVal: A New Measure of Work Values. Journal of Carreer Assessment, 22, 1-18. doi: 10.1177/1069072716639691

Duffy, R. D., Borges, N. J., \& Hartung, P. J. (2009). Personality, Vocational Interests, and Work Values of Medical Students. Journal of Career Assessment, 17(2), 189-200. doi: 10.1177/1069072708329035

Furnham, A., Petrides, K. V., Tsaousis, I., Pappas, K., \& Garrod, D. (2005). A cross-cultural investigation into the relationships between personality traits and work values. The Journal of Psychology: Interdisciplinary and Applied, 139(1), 5-31.

Hair, J. F., William, B., Babin, B., \& Anderson, R. E. (2009). Análise multivariada de dados ( $6^{\mathrm{a}}$ ed). Porto Alegre: Bookman.

Holland, J. L., \& Rayman, J. R. (1986). The SDS as an Innovation. In B, Walsh, \& S. H. Osipow (Eds.), Advances Vocational Psychology (vol. I - The Assessment of Interests) (55-81). London: Lawrence Erlbaum Associates.
Leuty, M. E., \& Hansen, J. C. (2011). Evidence of construct validity for work values. Journal of Vocational Behavior, 79, 379-390. doi: 10.1016/j.jvb.2011.04.008

Leuty, M. E., \& Hansen, J. C. (2013). Building Evidence of Validity: The Relation Between Work Values, Interests, Personality, and Personal Values. Journal of Career Assessment, 21(2), 175-189. doi: 10.1177/1069072712466714

Nunes, C. H. S. S. Hutz, C. S., \& Nunes, M. F. O. (2010). Bateria Fatorial de Personalidade (BFP): Manual técnico. São Paulo: Casa do Psicólogo.

Pinto, L. P., \& Noronha, A. P. P. (2014). Escala para Evaluación de los Valores de Trabajo (EAVT). Documento técnico no publicado. Campinas: Universidade São Francisco.

Primi, R; Mansão, C. M.; Muniz, M., \& Nunes, M. F. O. (2010). SDS-Questionário de Busca Autodirigida. Manual Técnico da versão brasileira. São Paulo: Casa do Psicólogo.

Rottinghaus, P. J., \& Zytowski, D. G. (2006). Commonalities between adolescents' work values and interests. Measurement and Evaluation in Counseling and Development, 38, 211-221.

Rounds, J., \& Armstrong, P. I. (2014). Integrating values and interests for career counseling. In M. Pope, L. Y. Flores, \& P. J. Rottinghaus (Eds.), The role of values in careers (pp. 101-113). Greensboro, NC: Information Age.

Rounds, J. B., \& Armstrong, P. I. (2005). Assessment of needs and values. In S. D. Brown \& R. W. Lent (Eds.), Career development and counseling (pp. 305-329). New York: John Wiley.

Savickas, M. L. (1999). The psychology of interests. In M. L. Savickas \& A. R. Spokane (Eds.), Vocational interests: Meaning, measurement, and counseling use (pp. 19-56). Palo Alto, CA: Davies-Black.

Super, D. E. (1957). The psychology of careers. New York: Harper \& Row.

Super, D. E. (1973). The Work Values Inventory. In D. G. Zytowski (Ed.), Contemporary apporaches to interest measurement (pp.189-205). Minneapolis: Univerity of Minneapolis Press.

Super, D. E. (1995). Values: their nature, assessment and practical use. In D. E. Super, \& B. Sverko (Eds.), Life roles, values, and careers: international findings of the Work Importance Study (pp.3-21). San Francisco: Jossey-Bass Publishers.

Sverko, B., Barbarovi, T., \& Sverko, I. (2008). Assessment of values and life salience. In J. A. Athanasou, \& R. Van Esbroeck (Eds.). International Handbook of Career Guidance (pp. 539-564). Dordrecht: Springer.

Sodano, S. M. (2011). Integrating work and basic values into the Spherical Model of Interests. Journal of Vocational Behavior, 78, 1-10. doi: 10.1016/j.jvb.2010.09.004

Sortheix, F. M., Chow, A., \& Salmela-Aro, K. (2015). Work values and the transition to work life: A longitudinal study. Journal of Vocational Behavior, 89, 162-171. doi: 10.1016/j.jvb.2015.06.001

Tien, H. L. S. (2011). An exploration of adult career interests and work values in Taiwan. Asia Pacific Education Review, 12, 559-568.

Vinken, H. (2007). New life course dynamics? Career orientations, work values and future perceptions of Dutch youth. Nordic Journal of Youth Research, 15(1), 9-30.

Para citar este artículo:

Pinto, L. P., Porto Noronha, A. P., \& Marín Rueda, F. J. (2017). Asociación entre valores de trabajo, intereses profesionales y rasgos de personalidad. Ciencias Psicológicas, 11(1), 57 - 65. doi: 10.22235/cp.v11i2.1347 